\title{
Semiparametric Mixed Models for Nested Repeated Measures Applied to Ambulatory Blood Pressure Monitoring Data
}

Rhonda D. Szczesniak

Division of Biostatistics \& Epidemiology, Division of Pulmonary Medicine, Cincinnati Children's Hospital Medical Center, rhonda.szczesniak@cchmc.org

Dan Li

Department of Mathematical Sciences, University of Cincinnati, lid7@mail.uc.edu

RaoufS. Amin

Division of Pulmonary Medicine, Cincinnati Children's Hospital Medical Center, raouf.amin@cchmc.org

Follow this and additional works at: http://digitalcommons.wayne.edu/jmasm

\section{Recommended Citation}

Szczesniak, Rhonda D.; Li, Dan; and Amin, Raouf S. (2016) "Semiparametric Mixed Models for Nested Repeated Measures Applied to Ambulatory Blood Pressure Monitoring Data," Journal of Modern Applied Statistical Methods: Vol. 15 : Iss. 1 , Article 14. DOI: $10.22237 /$ jmasm/1462075980

Available at: http://digitalcommons.wayne.edu/jmasm/vol15/iss1/14 


\section{Semiparametric Mixed Models for Nested Repeated Measures Applied to Ambulatory Blood Pressure Monitoring Data}

\section{Cover Page Footnote}

This work was partially supported by NHLBI/NIH Awards (Grant Numbers R01 HL080670 and M01 RR008084) and an Institutional Clinical and Translational Science Award, NIH/NCATS Grant Number 8UL1TR000077-05. Its contents are solely the responsibility of the authors and do not necessarily represent the official views of the NIH. 


\section{Semiparametric Mixed Models for Nested Repeated Measures Applied to Ambulatory Blood Pressure Monitoring Data}

\author{
Rhonda D. Szczesniak \\ Cincinnati Children's Hospital \\ Cincinnati, $\mathrm{OH}$
}

\author{
Dan Li \\ University of Cincinnati \\ Cincinnati, $\mathrm{OH}$
}

\author{
Raouf S. Amin \\ Cincinnati Children's Hospital \\ Cincinnati, $\mathrm{OH}$
}

Semiparametric mixed models are increasingly popular for statistical analysis of medical device studies in which long sequences of repeated measurements are recorded. Monitoring these sequences at different periods over time on the same individual, such as before and after an intervention, results in nested repeated measures (NRM). Covariance models to account for NRM and simultaneously address mean profile estimation with penalized splines via semiparametric regression are considered with application to a prospective study of 24-hour ambulatory blood pressure and the impact of surgical intervention on obstructive sleep apnea.

Keywords: functional data analysis, semiparametric regression, blood pressure, longitudinal data analysis, doubly repeated measures, obstructive sleep apnea, medical monitoring, circadian rhythm

\section{Introduction}

Medical device studies frequently involve collections of multiple recordings that result in long sequences of repeated measurements for each subject. It is often of interest to assess these sequences at different periods of time or recording sessions on the same subject. This type of data, commonly called nested repeated measures (NRM), yields two sources of intrasubject variation: an inner source arising from observations within a sequence and an outer source arising from observations under different time periods, such as before and after an intervention. Covariance models for NRM have been proposed to account for the intrasubject correlation arising from data of this nature (Harville, 1997; Laird \& Ware, 1982; Jennrich \& Schluchter, 1986). More recent work (Park \& Lee, 2002) shows covariate effects are impacted by the choice of covariance structure and a series of covariance

Dr. Szczesniak is an Associate Professor in the Division of Biostatistics \& Epidemiology. Email herat: rhonda.szczesniak@cchmc.org. 


\section{NESTED SEMIPARAMETRIC MIXED EFFECTS MODELS}

models to account for NRM can be specified in a linear mixed model. In these applications, mean profiles are modeled using polynomial regressions. The models are used to compare different experimental conditions or mean profiles by assessing overall mean differences.

For experiments in which the mean profile cannot be characterized with a parametric function, semiparametric mixed models may be useful. Penalized splines are a more flexible alternative to estimate the mean function (Eilers \& Marx, 1996), and can be expressed as the best linear unbiased prediction (BLUP) of a linear mixed model (Ruppert, Wand, \& Carroll, 2003, p. 99-100). Combining this nonparametric representation of the mean function with parametric estimation in the linear mixed model is referred to as semiparametric regression. Semiparametric mixed models have been used to compare the mean profiles of two independent groups (e.g. placebo versus treated) in a study of cardiovascular safety data (Maringwa et al., 2008a). Model selection was performed and mean profiles were estimated with linear penalized splines. The group-specific mean profiles were compared over time using simultaneous confidence bands. This approach, which has been used in other biomedical studies (see VanDyke et al., 2012 for an example), works well for single repeated measures factors. Semiparametric mixed models have also been applied to data arising from crossover designs to compare conditionspecific mean profiles over time (Maringwa et al., 2008b). In this study focused on crossover designs, correlation between and within periods were assumed to be separable (Jones \& Kenward, 2003, p. 193). This assumption corresponds to concluding that the outer repeated measures (between periods) may be accounted for by using subject-specific random intercepts. In NRM studies where outer repeated measures are collected at variable times across subjects, a more complex correlation structure may be necessary.

Despite inferential goals for time-specific comparisons that are similar to prior developments, the combination of NRM and an unrecognizable mean response function requires further methodological development for efficient regression parameter estimates. In this article, a series of semiparametric mixed models are proposed which incorporate NRM covariance modeling and mean profile estimation approaches. The following section begins with description of the ambulatory blood pressure monitoring data, which is the motivation for this development. Model selection criteria are then provided along with calculations for simultaneous confidence bands to assess time-specific intervention effects with application to the motivating data. The appropriateness of each proposed model for the data is discussed. Additional details on covariance models and relevant code are provided as Appendices. 


\section{SZCZESNIAK ET AL}

\section{Methodology}

\section{Twenty-Four Hour Ambulatory Blood Pressure Monitoring}

The motivating data arises from a prospective study to examine the effect of a surgical intervention on 24-hour ambulatory blood pressure. The importance of diurnal changes in blood pressure in predicting target organ damage has been demonstrated (Mansoor \& Massie, 1999). The application in this article focuses on diastolic blood pressure (DBP) profiles arising from ambulatory blood pressure monitoring. These profiles are recordings of systolic and diastolic blood pressures, observed in 30-minute intervals over a 24 -hour period beginning with time of sleep onset for each subject. In healthy subjects, these data typically have a marked circadian pattern with diurnal features that may not be present in subjects with obstructive sleep apnea (Mansoor, 2002). Previous analysis techniques have included the use of restricted cubic splines to fit DBP profiles in a study of hypertension during pregnancy (Lambert, Abrams, Jones, Halligan, \& Shennan, 2001).

This application focuses on whether DBP patterns change in subjects with obstructive sleep apnea after an intervention consisting of adenotonsillectomy. For the study, each subject wore an ambulatory blood pressure monitoring cuff at two separate recording sessions: before and after the intervention. The duration between baseline and follow-up ranged from six to twelve months and warranted consideration of more complex covariance models, as any level of improvement after intervention may be time-sensitive. Immediately prior to receiving the cuff to monitor blood pressure, demographic and clinical characteristics were obtained for each subject. Although monitoring data were equally spaced and collected both before and after the intervention for each of the 58 subjects, there were incomplete profiles due to cuff malfunctions that occurred during each 24-hour observation period. The timing of the follow-up monitoring and potential for incomplete profiles increase the importance of selecting an appropriate covariance model.

Figure 1 illustrates the pre- and post-surgical intervention profiles of five randomly selected subjects, demonstrating the intrasubject variability arising from the inner-repeated measures (within profiles) and the outer repeated measures (baseline and follow-up profiles), as well as the intersubject variability between profiles. Circadian rhythm in daytime and nighttime blood pressures is not apparent in the observed profiles, presumably due to the intra- and intersubject variation. In addition to assessing intervention effects on mean DBP response, the rate of change 


\section{NESTED SEMIPARAMETRIC MIXED EFFECTS MODELS}
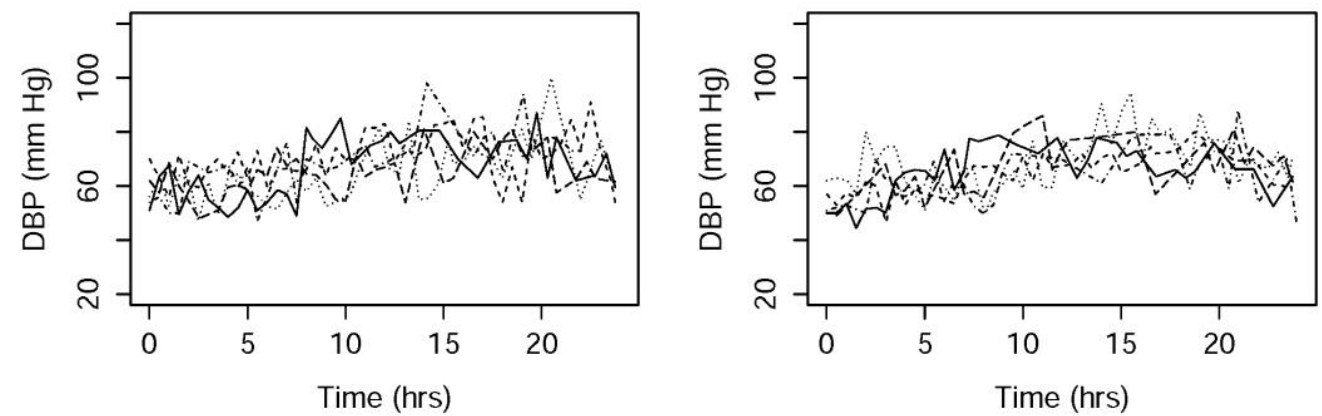

Figure 1. Subject-specific observed DBP response, before and after intervention

Five subject-specific profiles during recording of 24-hour diastolic blood pressure (DBP, $y$-axis) taken prior to surgery (left) and after surgery (right); $x$-axis represents time (in hours) since sleep onset

experienced by subjects when awakening from sleep (clinically termed the "morning blood pressure surge") is also of clinical importance. The presence of this feature indicates greater subject responsiveness to blood pressure regulation. Rates of change close to zero may indicate poor blood pressure control (Amin et al., 2008; Crisalli et al., 2012).

The statistical methodology in this manuscript relates to three inferential goals. First, develop an appropriate model for NRM covariance and spline representation of baseline and follow-up AMBP profiles. Second, determine how the rate of change or "morning surge" changes over time by using first-order derivatives of penalized regression splines. Third, construct simultaneous confidence bands to compare mean differences between baseline and follow-up AMBP profiles during daytime and nighttime.

\section{Modeling the Mean Response Function}

A penalized spline representation (Eilers \& Marx, 1996) is used to model the mean DBP response over the 24-hour interval. Ignoring intervention effect, this model can be expressed as

$$
\begin{aligned}
& \mathrm{DBP}_{i j k}=\mathrm{f}\left(t_{i j k}\right)+\varepsilon_{i j k}^{*}, \\
& \mathrm{f}\left(t_{i j k}\right)=\beta_{0}+\beta_{1} t_{i j k}+\ldots+\beta_{p} t_{i j k}^{p}+\sum_{l=1}^{L} b_{l}\left(t_{i j k}-\kappa_{l}\right)_{+}^{p}
\end{aligned}
$$




\section{SZCZESNIAK ET AL}

The term $\mathrm{DBP}_{i j k}$ represents the measurement of DBP for the $i^{\text {th }}$ subject $(i=1, \ldots, n)$ taken at the $j^{\text {th }}$ recording session $\left(j=1, \ldots, n_{i}\right)$ at time $t_{i j k}\left(k=1, \ldots, n_{i j}\right)$ during the 24-hour interval. The function $\mathrm{f}\left(t_{i j k}\right)$ is a combination of fixed effects parameters $\left(\beta_{0}, \beta_{1}, \ldots, \beta_{p}\right)^{\prime}$ and $p^{\text {th }}$ degree splines with knots at distinct locations $\left(\kappa_{1}, \ldots, \kappa_{L}\right)^{\prime}$ along the time interval with corresponding coefficients $\left(b_{1}, \ldots, b_{L}\right)^{\prime}$; assume that $b_{l} \sim \mathrm{N}\left(0, \sigma_{b}^{2}\right)$. Representations for the error term $\varepsilon_{i j k}^{*}$ are discussed in the context of the linear mixed model later in this section. A series of models similar to those from previous work using linear truncated power splines (Maringwa et al., 2008a) but expanded to incorporate NRM arise from (1) and may be considered to represent the overall shape of the 24-hour DBP, (Table 1). The knot locations are in the range of $t_{i j k}$ values, where $t_{+}=\max (0, t)$. To fit the mean function in our motivating example, quadratic $(p=2)$ penalized splines were used.

Structure (1.1) in Table 1 shows a common DBP curve for both pre- and postintervention, corresponding to no intervention effect. To assess whether the intervention effect is parallel, one can examine Structure (1.2). It is possible that post-intervention profiles have an average quadratic trend that differs from the trend during pre-intervention, without any changes to the more localized, nonparametric (spline) portion of the model. For this case, one can examine Structure (1.3). One can fit Structure (1.4) to capture more localized changes in average DBP features. The above distribution and independence assumptions hold in all model settings, except Structure (1.5.), which provides different degrees of smoothing based on whether the session occurred before or after intervention.

Semiparametric regression and NRM can be characterized in the familiar linear mixed model framework for longitudinal data (Verbeke \& Molenberghs, 2000, p. 23) as

$$
\mathbf{Y}_{i}=\mathbf{X}_{i} \boldsymbol{\beta}_{i}+\mathbf{Z}_{b} \mathbf{b}_{b}+\boldsymbol{\varepsilon}_{i}^{*}
$$

where $\mathbf{X}_{i}$ and $\boldsymbol{\beta}_{i}$ represent the traditional fixed effects design matrix and parameter vector, $\mathbf{Z}_{b}$ and $\mathbf{b}_{b}$ correspond to the previously-described design matrix for the spline basis function and coefficient vector, and the overall error vector $\boldsymbol{\varepsilon}_{i}{ }^{*}$ corresponds to the $\varepsilon_{i j k}^{*}$ in $(1) ; \boldsymbol{\varepsilon}_{i}^{*} \sim \mathrm{N}\left(0, \Sigma_{i}^{*}\right)$. The entire response for the $i^{\text {th }}$ subject, $\mathbf{Y}_{i}$, is an $n_{i} \times 1$ vector, where $n_{i \bullet}=\sum_{j=1}^{n_{i}} n_{i j}$. 


\section{NESTED SEMIPARAMETRIC MIXED EFFECTS MODELS}

Table 1. Mean response models for twenty-four hour diastolic blood pressure

\begin{tabular}{|c|c|}
\hline Effects Description & Mean Response Structure* \\
\hline (1.1) No intervention effect & $\beta_{0}+\beta_{1} t_{i j k}+\beta_{2} t_{i j k}^{2}+\sum_{l=1}^{L} b_{l}\left(t_{i j k}-K_{l}\right)_{+}^{2}$ \\
\hline $\begin{array}{l}\text { (1.2) Intervention effect constant } \\
\text { across 24-hour sequence }\end{array}$ & $\beta_{0}+\beta_{02}$ post $_{i j}+\beta_{1} t_{i j k}+\beta_{2} t_{i j k}^{2}+\sum_{l=1}^{L} b_{l}\left(t_{i j k}-K_{l}\right)_{+}^{2}$ \\
\hline $\begin{array}{r}\text { (1.3) Pre- and post- intervention } \\
\text { profiles have different quadratic } \\
\text { trends }\end{array}$ & $\begin{aligned} \beta_{0}+\beta_{02} \text { post }_{i j}+\beta_{1} t_{i j k}+\beta_{12} \text { post }_{i j} t_{i j k} & +\beta_{2} t_{i j k}^{2}+\beta_{12} \text { post }_{i j} t_{i j k}^{2} \\
& +\sum_{l=1}^{L} b_{l}\left(t_{i j k}-\kappa_{l}\right)_{+}^{2}\end{aligned}$ \\
\hline $\begin{array}{l}\text { (1.4) Pre- and post-intervention } \\
\text { profiles smoothed differently } \\
\text { using distinct vectors for } \\
\text { coefficients for pre- and post- } \\
\text { intervention profiles }\end{array}$ & $\begin{array}{r}\beta_{0}+\beta_{02} \text { post }_{i j}+\beta_{1} t_{i j k}+\beta_{12} \text { post }_{i j} t_{i j k}+\beta_{2} t_{i j k}^{2}+\beta_{12} \text { post }_{i j} t_{i j k}^{2} \\
+\sum_{l=1}^{L} b_{l}^{\text {pre }_{i j}}\left(t_{i j k}-\kappa_{l}\right)_{+}^{2}+\sum_{l=1}^{L} b_{l}^{\text {post }_{i j}}\left(t_{i j k}-\kappa_{l}\right)_{+}^{2}\end{array}$ \\
\hline $\begin{array}{r}\text { (1.5) Separate smoothing and } \\
\text { distinct smoothing parameters } \\
\text { for pre- and post-intervention } \\
\text { profiles }\end{array}$ & $\begin{array}{l}\text { Same as structure (1.4) but differing variances for smoothing } \\
\qquad \text { coefficients: } b_{l}^{\text {pre }_{i j}} \sim \mathrm{N}\left(0, \sigma_{b_{l}^{\text {pre }}}^{2}\right) \text { and } b_{l}^{\text {post }_{i j}} \sim N\left(0, \sigma_{b_{l}^{\text {post }}}^{2}\right)\end{array}$ \\
\hline
\end{tabular}

* The term post $i j$ refers to an indicator of post-intervention assessment ( 1 if observation taken during postintervention session, 0 otherwise); pre $i j$ is defined similarly for pre-intervention

The proposed structure provides flexibility for the covariance matrices, which may be advantageous for NRM. As described by Park and Lee (2002), the subjectspecific covariance matrix for the error term $\boldsymbol{\varepsilon}_{i}^{*}$ is

$$
\boldsymbol{\Sigma}_{i}^{*}=\left[\begin{array}{cccc}
\operatorname{Var}\left(\varepsilon_{i 1}^{*}\right) & \operatorname{Cov}\left(\varepsilon_{i 1}^{*}, \varepsilon_{i 2}^{*}\right) & \ldots & \operatorname{Cov}\left(\varepsilon_{i 1}^{*}, \varepsilon_{i n_{i}}^{*}\right) \\
\operatorname{Cov}\left(\varepsilon_{i 1}^{*}, \varepsilon_{i 2}^{*}\right) & \operatorname{Var}\left(\varepsilon_{i 2}^{*}\right) & \ldots & \operatorname{Cov}\left(\varepsilon_{i 2}^{*}, \varepsilon_{i n_{i}}^{*}\right) \\
\vdots & \vdots & \ddots & \vdots \\
\operatorname{Cov}\left(\varepsilon_{i 1}^{*}, \varepsilon_{i n_{i}}^{*}\right) & \operatorname{Cov}\left(\varepsilon_{i 1}^{*}, \varepsilon_{i 2}^{*}\right) & \ldots & \operatorname{Var}\left(\varepsilon_{i n_{i}}^{*}\right)
\end{array}\right]
$$

The $\operatorname{Var}\left(\varepsilon_{i j}^{*}\right)$ is the $n_{i} \times n_{i}$. variance-covariance matrix for the sequence of measurements from the $i^{\text {th }}$ subject observed on the $j^{\text {th }}$ occasion; $\operatorname{Cov}\left(\varepsilon_{i j}^{*}, \varepsilon_{i j^{\prime}}^{*}\right)$ for $j \neq j^{\prime}$ is the covariance matrix for measurements observed at distinct occasions $j$ and $j^{\prime}$. Different covariance models are now presented for the motivating example. 


\section{SZCZESNIAK ET AL}

\section{Covariance for Nested Repeated Measures}

Random effects: $\quad$ Random intercepts are one of the most commonly used methods to address intrasubject variability. In the case of a single repeated factor, subject-specific effects are often included as random intercepts and all measurements are assumed to have equal correlation. This assumption corresponds to compound symmetry. The approach can be naturally extended to NRM by including two additional random effects for occasion and sequence. For a given subject, any two measurements taken within a sequence during a single occasion have correlation $\rho_{s}$; two measurements taken at the same time point of sequences on two distinct occasions have correlation $\rho_{o}$. This covariance model has straightforward interpretation but may not be suited for many experiments with NRM. In the motivating DBP example, $\rho_{s}$ corresponds to an individual's measurements taken within a 24-hour period having the same correlation, regardless of the amount of time lapsing between measurements; the outer repeated measure correlation $\rho_{o}$ assumes that any two DBPs recorded at the same time during two separate 24-hour periods have a common correlation.

Composite covariance: Nonconstant correlation within the 24-hour period and unequal variances between visits are both plausible but neither can be addressed with the aforementioned random effects covariance structure. Instead, one can use a composite covariance model (Searle, 2006, p. 348) obtained using the right Kronecker product to model the sources of correlation arising from inner and outer repeated measures. There are several possibilities for composite covariance models, although there are some limitations imposed by software capabilities (Park \& Lee, 2002). For the DBP example, an unstructured covariance for the outer repeated measure (occasion) and $\mathrm{AR}(1)$ structure for the inner repeated measure (sequence) are considered. The covariance matrix corresponding to the direct product of unstructured and $\mathrm{AR}(1)$ covariance is 


\section{NESTED SEMIPARAMETRIC MIXED EFFECTS MODELS}

$$
\begin{aligned}
& {\left[\begin{array}{cc}
\sigma_{1}^{2} & \sigma_{21} \\
\sigma_{21} & \sigma_{2}^{2}
\end{array}\right] \otimes\left[\begin{array}{ccccc}
1 & \rho & \rho^{2} & \ldots & \\
\rho & 1 & \rho & \rho^{2} & \ldots \\
\rho^{2} & \rho & 1 & & \\
\vdots & \vdots & & \ddots & \\
& & & & 1
\end{array}\right]}
\end{aligned}
$$

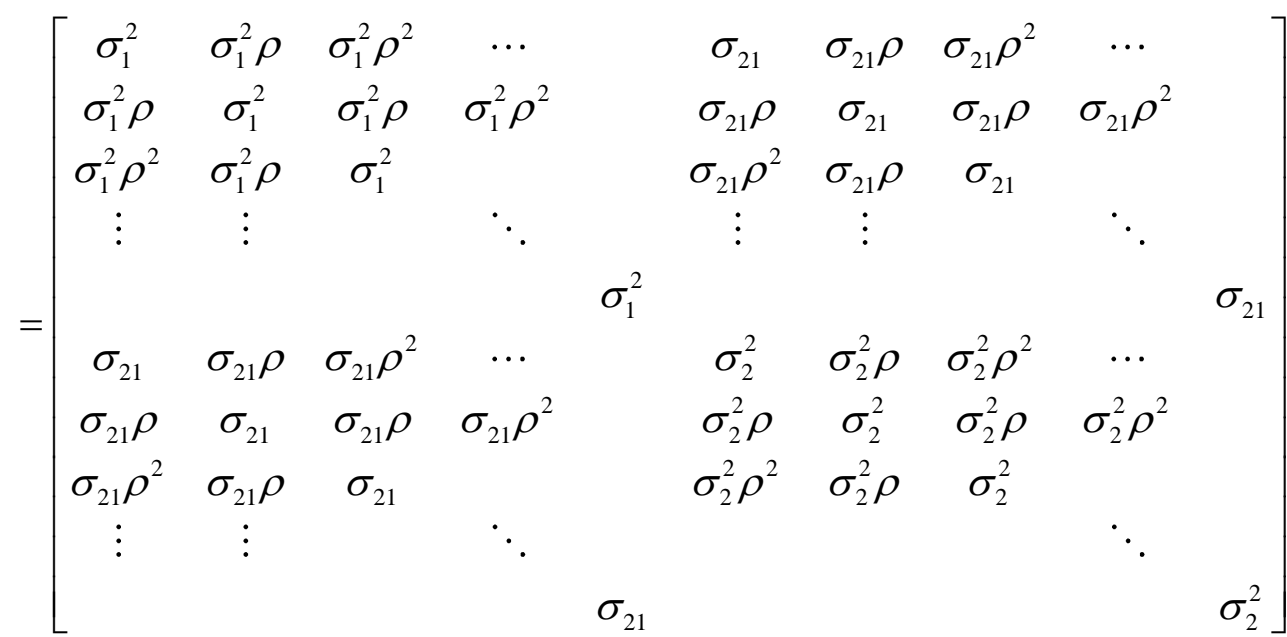

Exponential covariance: The composite covariance model assumes equally spaced measurement times within each occasion (at the sequence level) and between occasions. In the motivating example, the timing of post-intervention measurement is not equal across subjects, which suggests the need for a more flexible covariance model. The exponential covariance models described in this section have their origin in spatial statistics but can be used in the linear mixed model framework (Littell, Milliken, Stroup, Wolfinger, \& Schabenberger, 2006, p. 198) and have been used in various longitudinal data analysis applications with a single repeated measures factor (see Szczesniak et al., 2013 for a recent biomedical example). This section covers two general types of exponential covariance models.

Consider the semivariogram formula for the exponential covariance model with nugget effect:

$$
\mathrm{r}(h)=C_{n}+\sigma_{0}^{2}\left[1-\exp \left(-\frac{\left|t_{i j k}-t_{i j k^{\prime}}\right|}{a_{0}}\right)\right],
$$




\section{SZCZESNIAK ET AL}

where $\left|t_{i j k}-t_{i j k^{\prime}}\right|$ refers to the Euclidean distance between two time points, $t_{i j k}$ and $t_{i j k}$, thereby relaxing the previously-described assumption of equally-spaced inner repeated measures for the $\mathrm{AR}(1)$ covariance and accounting for irregular time series. The terms $C_{n}, C_{n}+\sigma_{0}^{2}$, and $a_{0}$ correspond to the geostatistical parameters referred to as the nugget, sill, and range (Wackernagel, 2003, p. 57). The nugget effect is a measure of the residual error or white noise of the DBP response; the range parameter dictates the decay of the covariance function. Please see Appendix A for a graphical explanation of these terms. If the term $C_{n}$ is excluded, then the model is considered an exponential covariance model without the nugget effect. Both versions of this exponential covariance model are applied to the motivating example.

\section{Results}

Fifty-eight subjects completed both recording sessions, and their observations comprise the data of interest. The median (Q1-Q3) time between pre- and postintervention measurement periods was 288.3 (218.1-321.5) days and ranged from as few as 177 days between visits to as much as 364 days between visits. This indicates the potential need to model unequally-spaced repeated measurements. The number of observed half-hourly DBP recordings over the 24-hour period was 41.0 (34.5-44.8) and 42.4 (36.0-46.2) at the baseline and follow-up sessions, respectively. Baseline age and BMI z-score were 9.0 (7.1-11.5) years and 1.47 (0.34-2.25), respectively; $40.4 \%$ of subjects were Caucasian and $46.8 \%$ were male.

The series of models from Table 1 were used to characterize DBP over the 24-hour sequence and the two measurement occasions. The mean response model chosen using adjusted fit statistics presented in previous work (Maringwa et al., 2008a) had Structure (1.3), which provided separate polynomial terms for each occasion but relied on the same smoothing parameter.

Each of the four previously described covariance functions was applied to model the correlation for the $\boldsymbol{\varepsilon}_{i}{ }^{*}$ term in (2). SAS code to implement the covariance models are in Appendix B. The estimates for the demographic covariates under each covariance model are presented in Table 2. Effect estimates were consistent across the four different covariance models, except for the effect of race, but this effect was not statistically significant in any of the models. Gender and BMI zscore were statistically significant in all models. 


\section{NESTED SEMIPARAMETRIC MIXED EFFECTS MODELS}

Table 2. Linear covariate effects* estimates from covariance models of diastolic blood pressure

\begin{tabular}{|c|c|c|c|c|}
\hline \multirow[b]{2}{*}{ Effect } & \multicolumn{4}{|c|}{ Covariance Model } \\
\hline & $\begin{array}{r}\text { Random Effects } \\
\text { Estimate (SE) } \\
p \text {-value }\end{array}$ & $\begin{array}{r}\text { Composite } \\
\text { Covariance } \\
\text { Estimate (SE) } \\
p \text {-value }\end{array}$ & $\begin{array}{r}\text { Exponential (with } \\
\text { nugget effect) } \\
\text { Estimate (SE) } \\
p \text {-value }\end{array}$ & $\begin{array}{r}\text { Exponential } \\
\text { (without nugget } \\
\text { effect) Estimate } \\
\text { (SE) } p \text {-value }\end{array}$ \\
\hline \multirow[t]{2}{*}{ Male } & $3.13970(1.10020)$ & $3.14310(1.11280)$ & $3.13940(1.12850)$ & $3.19640(1.10690)$ \\
\hline & 0.00640 & 0.00690 & 0.00780 & 0.00590 \\
\hline \multirow[t]{2}{*}{ Caucasian } & $-0.08742(1.09580)$ & $-0.08212(1.10810)$ & $-0.03037(1.12400)$ & $0.01099(1.10250)$ \\
\hline & 0.93680 & 0.94120 & 0.97860 & 0.99210 \\
\hline \multirow[t]{2}{*}{ Age (years) } & $0.18210(0.22670)$ & $0.19510(0.23100)$ & $0.18380(0.23670)$ & $0.17270(0.23080)$ \\
\hline & 0.42570 & 0.40210 & 0.44100 & 0.45780 \\
\hline Body Mass & $1.47780(0.48580)$ & $1.49850(0.51540)$ & $1.51340(0.56210)$ & $1.55500(0.52990)$ \\
\hline Index (z-score) & 0.00290 & 0.00460 & 0.00900 & 0.00440 \\
\hline
\end{tabular}

* These effects were assumed to enter the models linearly. Each model included the mean response function specified in Table 1, Structure (1.3)

\section{Evaluating Model Fit}

An important task in the model-building process is to select a suitable covariance structure. The effective number of parameters, referred to as $E_{p}$, can be obtained for each covariance model by estimating the appropriate covariance structure using (3). Let $\mathbf{C}=\left[\mathbf{X} \mathbf{Z}_{b}\right]$ be the design matrix for the mean response function and $\mathbf{B}=\left[\begin{array}{cc}0 & 0 \\ 0 & \mathbf{G}^{-1}\end{array}\right]$, where $\mathbf{G}$ corresponds to any random effects being used to model covariance (e.g. random intercepts), $\mathbf{R}=\operatorname{blkdiag}\left(\boldsymbol{\varepsilon}_{i}^{*}\right), i=1, \ldots, n_{i}$. The $E_{p}$ for each covariance model may be computed as: $E_{p}=\operatorname{trace}\left(\left(\mathbf{C}^{T} \mathbf{R}^{-1} \mathbf{C}+\mathbf{B}\right)^{-1} \mathbf{C}^{T} \mathbf{R}^{-1} \mathbf{C}\right)$.

Ultimately, the adjusted AIC can be computed as $\mathrm{AIC}_{\text {adj }}=-2 L L+2 E_{p}$. This calculation will take into account the additional parameters brought about by fitting the mean response function $\mathrm{f}(t)$ and the covariance function. SAS code for the $E_{p}$ calculations necessary for model structures in Table 1 and NRM covariance models is available from the authors upon request.

The fit statistics were calculated for each covariance model and are displayed in Table 3. The adjusted AIC and the more common information criteria (both marginal AIC and BIC) indicated that exponential covariance with a nugget effect provides the best fit of the covariance models considered. Subsequent estimation for the intervention effect is based on the exponential covariance with nugget. 


\section{SZCZESNIAK ET AL}

Table 3. Fit statistic results for covariance models

\begin{tabular}{rrrrrr} 
& \multicolumn{5}{c}{ Fit Statistics } \\
\cline { 2 - 6 } Covariance Model $^{*}$ & -2loglikelihood & AIC & BIC & Ep & AICadj \\
\hline Random Effects & 33494.0 & 33520.0 & 33494.0 & 12.1291 & 33518.3 \\
Composite Covariance & 33264.9 & 33296.9 & 33296.9 & 12.0024 & 33288.9 \\
Exponential (with nugget) & 32874.1 & 32904.1 & 32874.1 & 12.0604 & 32898.2 \\
Exponential (without nugget) & 33042.5 & 33070.5 & 33042.5 & 11.6404 & 33065.8 \\
\hline
\end{tabular}

* Each covariance model includes the mean response function specified in Table 1, Structure (1.3)
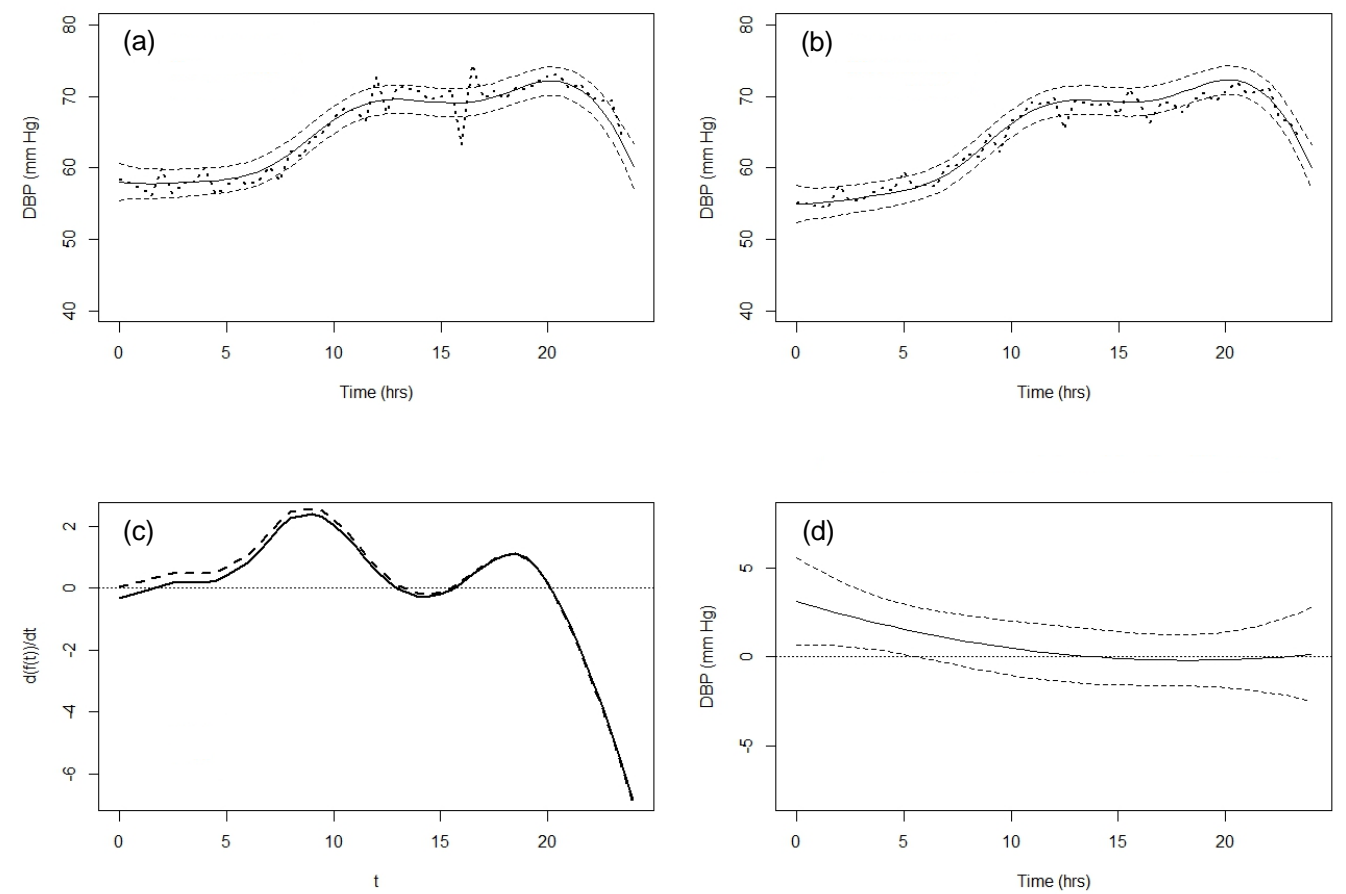

Figure 2. Fitted DBP response curves and morning surge, before and after intervention

The averaged response (jagged line), fitted curve (smooth, solid line) for $f(t)$ and corresponding $95 \%$ simultaneous confidence bands (dashed lines) during recording of the 24-hour diastolic blood pressure (DBP, $y$ axis) taken prior to surgery in (a) and after surgery in (b). The plot in (c) shows the derivative of the smooth function $f^{\prime}(t)$ for pre- and post-intervention sessions of 24-hour DBP recordings, where the solid (dashed) curve represents the rate of change for the pre-intervention (post-intervention) recordings. The difference between 24hour DBP mean response functions before and after intervention (solid line) and 95\% simultaneous confidence bands (dashed lines) are presented in (d). 


\section{NESTED SEMIPARAMETRIC MIXED EFFECTS MODELS}

Figure 2 (a-b) shows the fitted DBP curves corresponding to occasions before and after intervention, which both show smoother trends than the averaged response. The 95\% confidence bands accompanying each fitted curve are explained in a subsequent section. The circadian rhythm is more discernable here than examining the individual functions in Figure 1, and corresponds to previously mentioned studies that suggest a diurnal response over time. Nocturnal dipping is a feature that indicates healthier DBP rhythm. It is present at both occasions and is noticeable just before the sleep cycle starts for the next day (around $t=22$ hours after sleep onset).

The derivative $\mathrm{f}^{\prime}(t)$ of Structure (1.3) in Table 1 can be used to examine the morning surge before and after intervention. By looking at time since sleep onset, which corresponds to 0 on the $x$-axis in Figure 2 (c), the rate of change in average DBP is slightly higher for the intervention period; however, the derivative curves begin to overlap around $t=12$ hours after sleep onset. Presumably, 7-9 hours after sleep onset is the interval of interest to assess the morning surge, as this is the time frame when subjects begin to wake. Results suggest the rate of change is slightly elevated after intervention, as compared to before intervention. From a biomedical perspective, this finding may indicate heightened response to wakefulness as a result of receiving the intervention.

\section{Simultaneous Confidence Bands}

It is also of interest to examine the intervention effect on mean DBP response across the 24-hour interval. It is plausible to conduct point-by-point comparisons of the occasion-specific mean response functions. Rather than making this comparison of $\mathrm{f}_{\text {pre }}(t)$ to $\mathrm{f}_{\text {post }}(t)$ for all observed $t$ in the 24-hour interval, one can avoid those multiple comparison issues by constructing a simultaneous confidence band for this difference by using the following result (Ruppert et al., 2003, p. 142-143):

$$
\left[\begin{array}{c}
\hat{\boldsymbol{\beta}}-\boldsymbol{\beta} \\
\hat{\mathbf{b}}_{b}-\mathbf{b}_{b}
\end{array}\right] \sim \mathrm{N}\left\{\mathbf{0},\left(\mathbf{C}^{T} \mathbf{R}^{-1} \mathbf{C}+\mathbf{B}\right)^{-1}\right\}
$$

Define a grid $\mathbf{g}$ of time points $(0,23)$ by increments of 0.5 hours such that there are $T=49$ equally spaced time points $\left(g_{1}, \ldots, g_{49}\right)^{\prime}$. One can evaluate the estimated difference between the two functions as 


$$
\hat{\mathbf{f}}_{d}-\mathbf{f}_{d}=\left(\hat{\mathbf{f}}_{\text {pre }}-\hat{\mathbf{f}}_{\text {post }}\right)-\left(\mathbf{f}_{\text {pre }}-\mathbf{f}_{\text {post }}\right)=\mathbf{C}_{g}\left[\begin{array}{c}
\hat{\boldsymbol{\beta}}-\boldsymbol{\beta} \\
\hat{\mathbf{b}}_{b}-\mathbf{b}_{b}
\end{array}\right]=\left[\begin{array}{ll}
\mathbf{L X} & \mathbf{L} \mathbf{Z}_{b}
\end{array}\right]\left[\begin{array}{c}
\hat{\boldsymbol{\beta}}-\boldsymbol{\beta} \\
\hat{\mathbf{b}}_{b}-\mathbf{b}_{b}
\end{array}\right],
$$

where $\mathbf{L}=\left[\begin{array}{cccccc}1 & & & -1 & & \\ & \ddots & & & \ddots & \\ & & 1 & & & -1\end{array}\right]_{49 \times 98}$ is a contrast matrix, $\mathbf{X}$ and $\mathbf{Z}_{b}$ are the design matrices evaluated over $\mathbf{g}$, and $\mathbf{C}_{\mathbf{g}}=\left[\mathbf{L X} \mathbf{L Z}_{b}\right]$.

In order to obtain the $\operatorname{stdev}\left[\hat{\mathbf{f}}_{d}-\mathbf{f}_{d}\right]$ for the confidence band, it is necessary to compute $\operatorname{Cov}\left[\hat{\mathbf{f}}_{d}-\mathbf{f}_{d}\right]$. Using the following result:

$$
\operatorname{Cov}\left(\left[\begin{array}{c}
\hat{\boldsymbol{\beta}}-\boldsymbol{\beta} \\
\mathbf{b}_{b}-\mathbf{b}_{b}
\end{array}\right]\right)=\left(\mathbf{C}^{T} \mathbf{R}^{-1} \mathbf{C}+\mathbf{B}\right)^{-1}
$$

one can obtain a $95 \%$ simultaneous confidence band for $\mathrm{f}_{d}$ as

$$
\left[\hat{\mathrm{f}}_{d} \pm h_{0.95} \operatorname{stdev}\left\{\hat{\mathrm{f}}_{d}-\mathrm{f}_{d}\right\}\right]_{1 \leq l \leq T}
$$

where $h_{0.95}$ is the $1-\alpha$ quantile with $\alpha=0.05$. Finally, it can be approximated as

$$
\sup \left|\frac{\hat{\mathbf{f}}_{d}-\mathbf{f}_{d}}{\operatorname{stdev}\left\{\hat{\mathbf{f}}_{d}-\mathbf{f}_{d}\right\}}\right| \approx \max _{1 \leq l \leq T}\left|\frac{\left(\mathbf{C}_{g}\left[\begin{array}{c}
\hat{\boldsymbol{\beta}}-\boldsymbol{\beta} \\
\hat{\mathbf{b}}_{b}-\mathbf{b}_{b}
\end{array}\right]\right)_{l} \mid}{\operatorname{stdev}\left\{\hat{\mathbf{f}}_{d}-\mathbf{f}_{d}\right\}}\right|
$$

As an example, if simulations from (4) then computations of (5) are repeated 10,000 times, then the value of the ranked 9,500 th quantity is used as $h_{0.95}$. Similarly, a $95 \%$ pointwise confidence band for $\mathrm{f}_{d}$ is

$$
\left[\hat{\mathrm{f}}_{d} \pm Z_{0.95} \operatorname{stdev}\left\{\hat{\mathrm{f}}_{d}-\mathrm{f}_{d}\right\}\right]_{1 \leq l \leq T}
$$




\section{NESTED SEMIPARAMETRIC MIXED EFFECTS MODELS}

where $Z_{0.95}=1$.96. SAS code for both simultaneous and pointwise confidence bands is available from the authors upon request.

The simultaneous confidence band for $\mathbf{f}_{d}$ in the DBP example is plotted in Figure 2 (d). Portions of the band that do not overlap with zero on the $y$-axis are considered to indicate statistically significant differences between pre- and postintervention periods. As expected, the significant differences occur roughly for the first 5 hours of sleep (from $t=0$ to $t=5$ hours) and indicate mean DBP lowers after intervention. The mean profiles otherwise show substantial overlap, particularly during daytime ( $t>10$ hours). If there is any effect from intervention, it likely occurs during nighttime. Although not shown here, pointwise confidence bands for $\mathbf{f}_{d}$ indicate similar findings but have narrower bands because there is no adjustment for simultaneity.

\section{Conclusion}

With so many devices offering the opportunity to measure real-time subject outcomes over extended periods of time, many researchers may be overwhelmed by the amount of data and the task of determining an appropriate statistical method to assess treatment effects. Extending semiparametric mixed models to account for NRM offers a solution to such challenges. In the motivating example with 24-hour DBP recording, using this approach shows that intervention effects may be observable during sleep. It is likely that these findings would be masked if one tests summary measures from the DBP curves. Incorporating penalized splines provided a more sensitive means to assess medically important features of the DBP profile, such as nocturnal dipping and morning surge. Findings using semiparametric regression suggest the presence of an unexpected "daytime dip." These findings are not consistent with the DBP profiles of healthy controls but reflect prior studies of rough averages of DBP over the 24-hour period (Amin et al., 2008).

Ignoring the impact from NRM on the regression model reduces efficiency in the parameter estimates and may lead to incorrect conclusions about intervention effects. Analyses of the DBP data show improvement in model fit is attributable to accounting for unequally spaced measurement times. There are other covariance models that can also be implemented in the SAS MIXED procedure to account for NRM. Some examples include the Gaussian covariance model (nugget effect specification is optional) and the right Kronecker product "AR(1)®UN," which corresponds to the DBP data to having AR(1) covariance for the outer repeated

measure (occasion) and unstructured covariance for the inner repeated measure (sequence). The authors attempted to fit these covariance models to the data but 


\section{SZCZESNIAK ET AL}

estimates were not obtained due to convergence issues from the MIXED procedure. An alternative approach to the semiparametric mixed models presented here is to perform sequential or hierarchical regression via path analysis (Snijders, 1996). Additional consideration in the model setup would be needed to incorporate the spline basis functions at subject-specific levels.

There are several ways in which the semiparametric mixed model with NRM covariance presented here can be further explored and extended. Functional principal components analysis may be used to examine dominant modes of variation in the subject- and visit-specific DBP profiles (Silverman, 1996); recent developments have been made to apply this approach on NRM (Shou, Zipunniokov, Crainiceanu, \& Greven, 2014). If data have a mean response function with sharp changes, multiple knots may be desirable in that region, and it may be advantageous to change knot locations of sequences observed at different periods. For such instances, adaptive spline methods may be useful; however, some methods may require different estimation approaches (DiMatteo, Genovese, \& Kass, 2001). It may also be of interest to assess the correlation between spline coefficients for the difference between occasions. Clinical and demographic characteristics in this study were assumed to enter the model linearly as covariates, but that assumption may be relaxed using generalized additive models (Hastie \& Tibshirani, 1990, p. 136-171). Although not the focus of this work, missing data is a pervasive issue. The approach used in this study essentially assumed the missing mechanism was MAR (Rubin, 1976); however, more recent work has been done to improve efficiency of estimators in semiparametric regression models in the presence of missing data ( $\mathrm{Yu} \& \mathrm{Nan}, 2006$ ). That work may be extended to the NRM covariance models presented here.

\section{References}

Amin, R., Somers, V. K., McConnell, K., Willging, P., Myer, C., Sherman, M., McPhail, G. L., Morgenthal, M., Fenchel, M. C., Bean, J., Kimball, T., \& Daniels, S. (2008). Activity-adjusted 24-hour ambulatory blood pressure and cardiac remodeling in children with sleep disordered breathing. Hypertension, 51(1), 84-91. doi: 10.1161/HYPERTENSIONAHA.107.099762

Crisalli, J. A., McConnell, K., VanDyke, R. D., Fenchel, M. C., Somers, V. K., Shamsuzzaman, A., Chini, B., Daniels, S. R., \& Amin, R. (2012). Baroreflex sensitivity after adenotonsillectomy in children with obstructive sleep apnea during wakefulness and sleep. Sleep, 35(10),1335-1343. doi: 10.5665/sleep.2108 


\section{NESTED SEMIPARAMETRIC MIXED EFFECTS MODELS}

DiMatteo, I., Genovese, C. R., \& Kass, R. E. (2001). Bayesian curve-fitting with free-knot splines. Biometrika, 88(4), 1055-1071. doi:

10.1093/biomet/88.4.1055

Eilers, P. H. C., \& Marx, B. D. (1996). Flexible smoothing with B-splines and penalties. Statistical Science, 11(2), 89-102. doi: 10.1214/ss/1038425655

Harville, D. A. (1977). Maximum likelihood approaches to variance component estimation and to related problems. Journal of the American Statistical Association, 72(358), 320-338. doi: 10.1080/01621459.1977.10480998

Hastie, T. J., \& Tibshirani, R. J. (1990). Generalized additive models. Monographs on statistics and applied probability. Boca Raton, FL: Chapman \& Hall/CRC.

Jennrich, R. I., \& Schluchter, M. D. (1986). Unbalanced repeated-measures models with structured covariance matrices. Biometrics, 42(4), 805-820. doi: $10.2307 / 2530695$

Jones, B., \& Kenward, M. G. (2003). Design and analysis of cross-over trials (2nd ed.). Monographs on statistics and applied probability. Boca Raton, FL: Chapman \& Hall/CRC.

Laird, N. M., \& Ware, J. H. (1982). Random-effects models for longitudinal data. Biometrics, 38(4), 963-974. doi: 10.2307/2529876

Lambert, P. C., Abrams, K. R., Jones, D. R., Halligan, A. W., \& Shennan, A. (2001). Analysis of ambulatory blood pressure monitor data using a hierarchical model incorporating restricted cubic splines and heterogeneous within-subject variances. Statistics in Medicine, 20(24), 3789-3805. doi: 10.1002/sim.1172

Littell, R. C., Milliken, G. A., Stroup, W. W., Wolfinger, R. D., \& Schabenberger, O. (2006). SAS for mixed models. Cary, NC: SAS Institute, Inc.

Mansoor, G. A. (2002). Sleep actigraphy in hypertensive patients with the 'non-dipper' blood pressure profile. Journal of Human Hypertension, 16(4), 237 242. doi: $10.1038 /$ sj.jhh.1001383

Mansoor, G. A., \& Massie, B. M. (1999). Left ventricular hypertrophy: A potent cardiovascular risk factor and its relationship to office and ambulatory blood pressure. Blood Pressure Monitoring, 4(Suppl 1), S19-S22.

Maringwa, J. T., Geys, H., Shkedy, Z., Faes, C., Molenberghs, G., Aerts, M., Ammel, K. V., Teisman, A., \& Bijnens, L. (2008a). Application of semiparametric mixed models and simultaneous confidence bands in a cardiovascular safety experiment with longitudinal data. Journal of 


\section{SZCZESNIAK ET AL}

Biopharmaceutical Statistics, 18(6), 1043-1062. doi:

10.1080/10543400802368881

Maringwa, J. T., Geys, H., Shkedy, Z., Faes, C., Molenberghs, G., Aerts, M., Ammel, K. V., Teisman, A., \& Bijnens, L. (2008b). Analysis of cross-over designs with serial correlation within periods using semi-parametric mixed models. Statistics in Medicine, 27(28), 6009-6033. doi: 10.1002/sim.3363

Ngo, L., \& Wand, M. P. (2004). Smoothing with Mixed Model Software. Journal of Statistical Software, 9(1), 1-54. Retrieved from http://ro.uow.edu.au/eispapers/2649/

Park, T., \& Lee, Y. J. (2002). Covariance models for nested repeated measures data: Analysis of ovarian steroid secretion data. Statistics in Medicine, 21(1), 143-164. doi: 10.1002/sim.949

Rubin, D. (1976). Inference and missing data. Biometrika, 63(3), 581-592. doi: 10.1093/biomet/63.3.581

Ruppert, D., Wand, M. P., \& Carroll, R. J. (2003). Semiparametric regression. Cambridge series in statistical and probabilistic mathematics. Cambridge; NY: Cambridge University Press.

Searle, S. R. (2006). Matrix algebra useful for statistics. Wiley series in probability and statistics. Hoboken, NJ: Wiley-Interscience.

Shou, H., Zipunniokov, V., Crainiceanu, C. M., \& Greven, S. (2014). Structured functional principal component analysis. Biometrics, 71(1), 247-257. doi: 10.1111/biom.12236

Silverman, B. W. (1996). Smoothed functional principal components analysis by choice of norm. The Annals of Statistics, 24(1), 1-24. doi:

10.1214/aos/1033066196

Snijders, T. (1996). Analysis of longitudinal data using the hierarchical linear model. Quality and Quantity, 30(4), 405-426. doi: 10.1007/BF00170145

Szczesniak, R. D., McPhail, G. L., Duan, L. L., Macaluso, M., Amin, R. S., \& Clancy, J. P. (2013). A semiparametric approach to estimate rapid lung function decline in cystic fibrosis. Annals of Epidemiology, 23(12), 771-777. doi: 10.1016/j.annepidem.2013.08.009

VanDyke, R. D., Ren, Y., Sucharew, H. J., Miodovnik, M., Rosenn, B., \& Khoury, J. C. (2012). Characterizing maternal glycemic control: a more informative approach using semiparametric regression. The Journal of MaternalFetal \& Neonatal Medicine, 25(1), 15-19. doi: 10.3109/14767058.2012.626922 


\section{NESTED SEMIPARAMETRIC MIXED EFFECTS MODELS}

Verbeke, G., \& Molenberghs, G. (2000). Linear mixed models for longitudinal data. Springer series in Statistics. New York, NY: Springer.

Wackernagel, H. (2003). Multivariate geostatistics: an introduction with applications (3rd ed.). Berlin, Germany: Springer.

Yu, M., \& Nan, B. (2006). A revisit of semiparametric regression models with missing data. Statistica Sinica, 16(4), 1193-1212. Retrieved from

http://www3.stat.sinica.edu.tw/statistica/J16N4/J16N46/J16N46.html 


\section{Appendix A: Semiovariogram Description}

Revisiting the exponential covariance model from the Methodology section, the semivariogram is:

$$
\mathrm{r}(h)=C_{n}+\sigma_{0}^{2}\left[1-\exp \left(-\frac{|h|}{a_{0}}\right)\right], \quad|h|>0
$$

The parameters $C_{n}, C_{n}+\sigma_{0}^{2}$, and $a_{0}$ correspond to geostatistical parameters: nugget, sill, and range. The covariance model with $\operatorname{var}(\varepsilon)=C_{n}+\sigma_{0}^{2}$ is called an exponential model with a nugget effect, whereas the covariance model with $\operatorname{var}(\varepsilon)=\sigma_{0}^{2}$ is called no-nugget effect model. In a nugget model, $\sigma_{0}^{2}$ is the partial sill (see Figure A1 below).

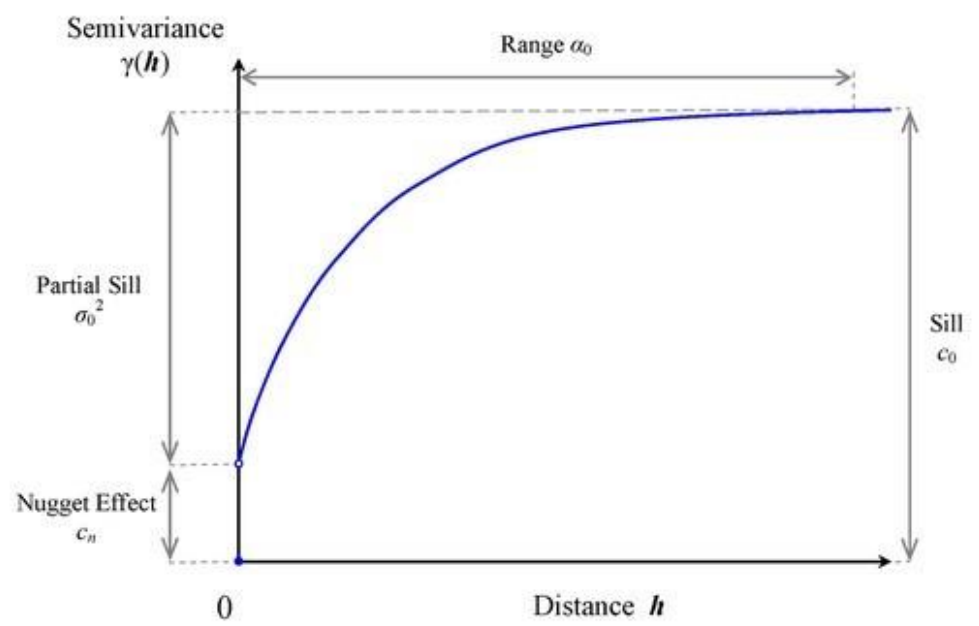

Figure A1. Semivariogram of exponential covariance

\section{Appendix B: SAS Implementation}

Covariance models (a-d) are presented for the four distinct variance-covariance matrices discussed in the paper, assuming mean response with Structure (1.3) from Table 1. Model structures (1.1-1.5) from Table 1 of the paper may be obtained using the approach described by Maringwa et al. (2008a) but assuming the selected 


\section{NESTED SEMIPARAMETRIC MIXED EFFECTS MODELS}

covariance function for the DBP data (exponential covariance with nugget effect). Models implemented in SAS are indexed below as 1.3(a-d).

Table B1. Description of variables used in SAS

\begin{tabular}{|c|c|}
\hline Variable & Description \\
\hline DBP & Response variable, diastolic blood pressure \\
\hline Visit & Occasion of measurement (either pre- or post-intervention) \\
\hline Studynr & Subject id for the study \\
\hline NTime & Time of DBP measurement since sleep onset (in hours) \\
\hline Timesq & Squared value of NTime \\
\hline Gender & Indicator variable for gender $(1=$ Male, $0=$ Female $)$ \\
\hline Bi_race & Indicator variable for race $(1=$ White, $0=$ Non-white $)$ \\
\hline$\overline{B M I Z}$ & Continuous variable representing BMI z-score from CDC \\
\hline NTimecat & Duplicate variable of NTime created for class statement \\
\hline Z1-Z15 & Columns of $Z$ matrix (quadratic) for smoothing ( $K=15$ knots) \\
\hline
\end{tabular}

Knots were selected using the algorithm from Ngo and Wand (2004). There were 15 knots, ranging from 2.54 to 22.97 hours since sleep onset.

\section{Model 1.3a: Random intercepts}

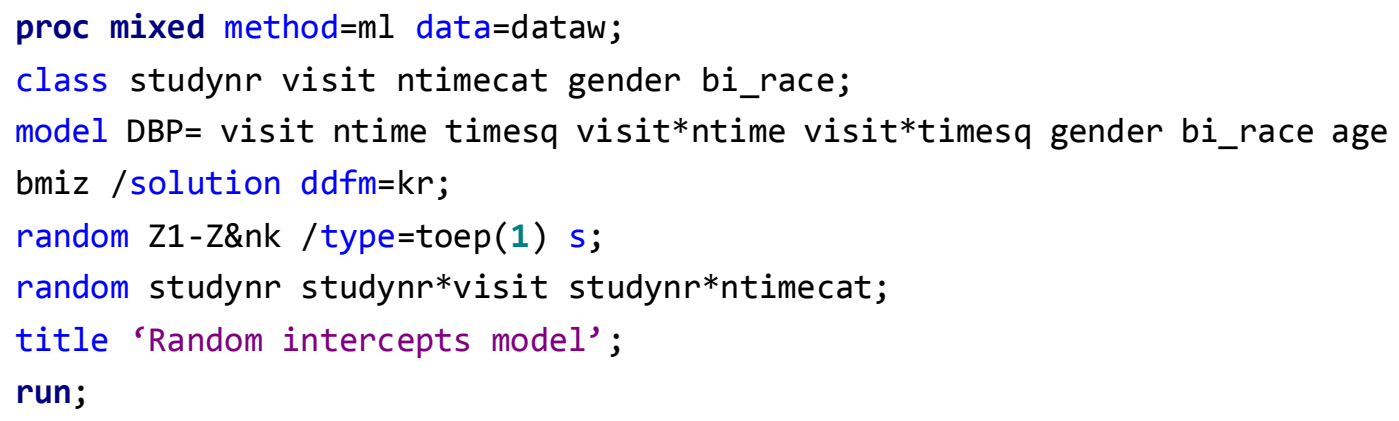

\section{Model 1.3b: Composite covariance}

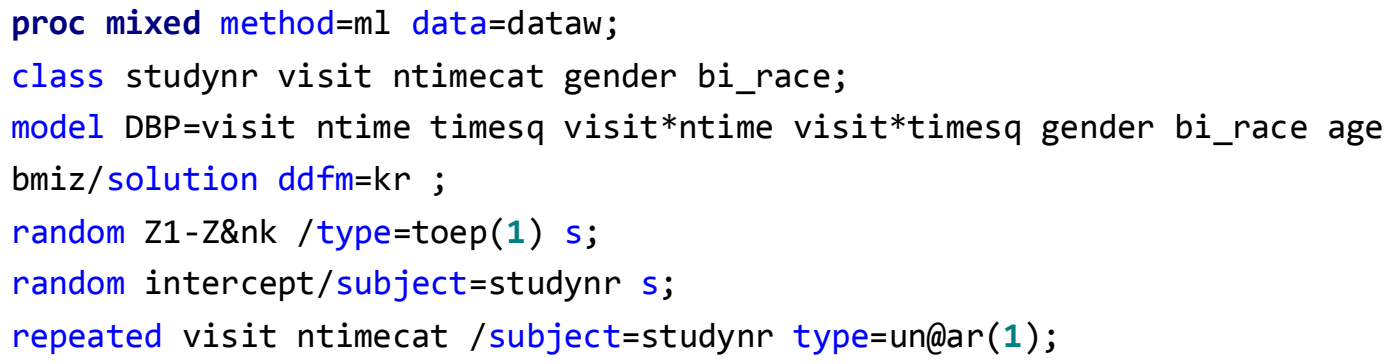




\section{SZCZESNIAK ET AL}

title 'AR(1)@UN composite covariance';

run;

\section{Model 1.3c: Exponential covariance (with nugget)}

proc mixed method=ml data=dataw convf;

class studynr visit gender bi_race;

model $\mathrm{DBP}=\mathrm{visit}$ ntime timesq visit*ntime visit*timesq gender bi_race age $\mathrm{bmiz} / \mathrm{ddfm}=\mathrm{kr} \mathrm{s}$;

random Z1-Z\&nk /type=toep(1) s;

random intercept/subject=studynr s;

repeated/subject=studynr type=sp(exp) (ntime_all) local;

title 'SP(EXP) Covariance (with nugget)';

run;

\section{Model 1.3d: Exponential covariance (without nugget)}

proc mixed method=ml data=dataw convf;

class studynr visit gender bi_race;

model $\mathrm{DBP}=$ visit ntime timesq visit*ntime visit*timesq gender bi_race age bmiz/ddfm=kr solution;

random Z1-Z\&nk /type=toep(1) s;

random intercept/subject=studynr s;

repeated/subject=studynr type=sp (exp) (ntime_all);

title 'SP(EXP) Covariance (without nugget)';

run; 